\title{
Realization of 2D Spin-Orbit Interaction and Exotic Topological Orders in Cold Atoms
}

\author{
Xiong-Jun Liu, ${ }^{1,2}$ K. T. Law, ${ }^{1}$ and T. K. $\mathrm{Ng}^{1}$ \\ ${ }^{1}$ Department of Physics, Hong Kong University of Science and Technology, Clear Water Bay, Hong Kong, China \\ ${ }^{2}$ Institute for Advanced Study, Hong Kong University of Science and Technology, Clear Water Bay, Hong Kong, China
}

(Received 10 April 2013; revised manuscript received 24 July 2013; published 24 February 2014)

\begin{abstract}
A Majorana zero bound mode exists in the vortex core of a chiral $p+i p$ superconductor or superfluid, which can be driven from an $s$-wave pairing state by two-dimensional spin-orbit coupling. We propose here a novel scheme based on realistic cold atom platforms to generate two-dimensional spin-orbit interactions in a blue-detuned square optical lattice, and predict both the quantum anomalous Hall effect and chiral topological superfluid phase in the experimentally accessible parameter regimes. This work may open a new direction with experimental feasibility to observe non-Abelian topological orders in cold atom systems.
\end{abstract}

DOI: 10.1103/PhysRevLett.112.086401

PACS numbers: 71.10.Pm, 03.65.Vf, 37.10.Jk, 67.85.Lm

The search for non-Abelian Majorana fermions has been a focus of both theoretical and experimental studies in condensed matter physics, driven by both the pursuit of exotic fundamental physics and the applications in faulttolerant topological quantum computation [1-3]. Majorana zero modes (MZMs) are predicted to exist in the vortex core of a two-dimensional (2D) intrinsic ( $p+i p)$-wave superconductor (SC) and at the ends of a one-dimensional (1D) $p$-wave SC [1]. Recent studies show that 1D and 2D effective $p$-wave superconductivity can be obtained through heterostructures formed by conventional $s$-wave SCs and topological insulators [4] or spin-orbit (SO) coupled semiconductors with Zeeman splitting [5,6], leading to MZMs in the case of an odd number of subbands crossing the Fermi level. Following the theoretical prediction, signatures of Majorana end states have been observed in the semiconductor nanowire or $s$-wave superconductor heterostructures [7] through tunneling transport measurements [8]. However, demonstrating the non-Abelian statistics of MZMs in the braiding operation [2], as an unambiguous verification, is a far more demanding task and not yet available in solid-state experiments.

On the other hand, the recent great advancement in realizing synthetic SO coupling in cold atoms [9-14] opens intriguing new avenues to probe topological phases [15-28] with clean platforms in a fully controllable fashion. So far the experimentally realized SO interaction [10-14] is a 1D SO term with equal Rashba and Dresselhaus amplitudes through a two-photon Raman process as theoretically pointed out in Ref. [9]. Detailed investigations show that only the 1D SO term can be realized in an atom gas in the continuum with an internal $\Lambda$-type configuration as considered in experiments $[9,29,30]$. With such $1 \mathrm{D}$ SO interaction it is unfortunately not optimistic to reach the topological superfluid (SF) phase from an $s$-wave pairing state in cold atoms. The reason is because, on the one hand, in a quasi-1D system no long-range $s$-wave order can be obtained; on the other hand, the proximity effect, as used in solid-state experiments [7], is not realistic for cold atoms. Therefore, to find out a truly experimental scheme to observe MZMs with higher dimensional SO interactions is a foremost outstanding goal in the current field of cold atoms.

In this Letter, we propose a novel scheme to observe 2D SO interaction for (pseudo)spin- $1 / 2$ cold fermions trapped in a blue-detuned square optical lattice and coupled to two periodic Raman fields, and predict the quantum anomalous Hall (QAH) effect and chiral topological SF phase in the single-particle and interacting regimes, respectively. The main results of this Letter include several important aspects. (I) The realization of the 2D SO interaction is based on the minimal setup by integrating the creations of a bluedetuned square lattice and periodic Raman fields though the same standing-wave lasers. The minimal realization is topologically stable against phase fluctuations of the applied lasers. (II) In the single-particle regime, the realized 2D SO coupled system is already nontrivial and describes a QAH insulator, and in the interacting regime the topological SF phase with MZMs can be obtained. (III) With the numerical estimates based on the realistic cold atom $\left({ }^{40} \mathrm{~K}\right)$ systems, we show that the predicted SO interaction and exotic topological orders can be studied with currently available experimental platforms.

We start with the quasi-2D cold fermions trapped in a conventional square optical lattice, with their internal degree of freedom (atomic spins) experiencing periodic Raman fields $M_{x, y}$ induced by two-photon processes, as illustrated in Fig. 1. The dynamics of fermions are governed by the following Hamiltonian:

$$
\begin{aligned}
H= & \frac{p_{x}^{2}}{2 m}+\frac{p_{y}^{2}}{2 m}+V(\mathbf{r})+m_{z}\left(\left|g_{\uparrow}\right\rangle\left\langle g_{\uparrow}|-| g_{\downarrow}\right\rangle\left\langle g_{\downarrow}\right|\right) \\
& -\left\{\left[M_{x}(x)+i M_{y}(y)\right]\left|g_{\uparrow}\right\rangle\left\langle g_{\downarrow}\right|+\text { H.c. }\right\},
\end{aligned}
$$

where $M_{x}=M_{0} \sin \left(k_{0} x\right)$ and $M_{y}=M_{0} \sin \left(k_{0} y\right)$, and the optical-dipole potential $V(\mathbf{r})=-V_{0}\left[\cos ^{2}\left(k_{0} x\right)+\cos ^{2}\left(k_{0} y\right)\right]$ forms the square lattice. Below we describe how to obtain 


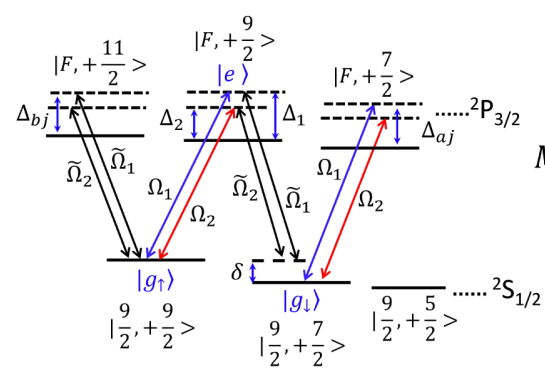

(a)

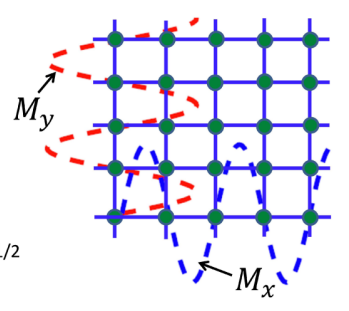

(b)
FIG. 1 (color online). (a) A realistic optical-dipole transition diagram in cold fermions $\left({ }^{40} \mathrm{~K}\right)$ coupled to two pairs of laser beams $\Omega_{1,2}$ (along the $x, y$ directions, respectively) and $\tilde{\Omega}_{1,2}$ (along the $z$ direction) under the blue-detuned condition. The notations F, P, S are angular momentum indices for atomic states. (b) With this configuration, a square lattice potential and two periodic Raman fields $M_{x, y}$ are simultaneously generated.

$H$ with the realistic cold atom platform (details of the realization can be found in the Supplemental Material [31]). The diagram for light-atom couplings is shown in Fig. 1(a), where four blue-detuned lasers are applied to induce the couplings, with two standing-wave lasers $\Omega_{1,2}$ linearly polarized along the $z$ axis and propagating in the $x-y$ plane, and two $\sigma_{+}$-polarized plane-wave lasers $\tilde{\Omega}_{1,2}$ propagating along the $z$ direction. According to the selection rule, the standing-wave and plane-wave lasers can induce the transitions from ground states $\left|g_{\uparrow, \downarrow}\right\rangle$ to excited ones depicted by the blue (for $\Omega_{1}$ ), red (for $\Omega_{2}$ ), and black (for $\tilde{\Omega}_{1,2}$ ) lines in Fig. 1(a), respectively. For the present purpose, we set $\Omega_{1}=\Omega_{0} \sin \left(k_{0} x\right)$ and $\Omega_{2}=\Omega_{0} \sin \left(k_{0} y\right)$, while $\tilde{\Omega}_{1}=i \tilde{\Omega}_{2}=\Omega_{0}$ are constants [31]. In the parameter regime that $\Delta_{1,2} \gg\left|\Delta_{1}-\Delta_{2}\right| \gg\left|\Omega_{0}^{2} / \Delta_{1,2}\right|$, the population of the excited state $|e\rangle$ is negligible [9], and the effective model of ground states is governed by following two effects induced by these transitions. First, the atom-light couplings contribute to diagonal potentials for $\left|g_{\uparrow, \downarrow}\right\rangle$ given by $V_{\uparrow}=$ $\sum_{j=1,2} \hbar\left(\left|\Omega_{j}\right|^{2} / \Delta_{j}+\left|\tilde{\Omega}_{j}\right|^{2} / \Delta_{b_{j}}\right)$ and $V_{\downarrow}=\sum_{j} \hbar\left(\left|\tilde{\Omega}_{j}\right|^{2} / \Delta_{j}+\right.$ $\left.\left|\Omega_{j}\right|^{2} / \Delta_{a_{j}}\right)$, which create the square lattice. Second, note that the couplings between $\left|g_{\uparrow, \downarrow}\right\rangle$ and $|e\rangle$ consist of two $\Lambda$-type configurations, through which two independent Raman fields are generated. In particular, $\Omega_{1}, \tilde{\Omega}_{1}$ generate one Raman field $M_{x}=\hbar\left|\Omega_{0}\right|^{2} \sin \left(k_{0} x\right) / \Delta_{1}$, and $\Omega_{2}, \tilde{\Omega}_{2}$ generate another one given by $i M_{y}=i \hbar\left|\Omega_{0}\right|^{2} \sin \left(k_{0} y\right) / \Delta_{2}$. The Raman processes can be precisely tuned in experiment to have a small two-photon off resonance $\left.\delta\left(|\delta| \ll\left|\Omega_{0}^{2} / \Delta_{1,2}\right|\right)\right)$ which gives rise to the constant Zeeman term with $m_{z}=$ $\hbar \delta / 2$ in $H$ [19]. We note that while the applied lasers $\Omega_{1,2}$ and $\tilde{\Omega}_{1,2}$ can also couple to other ground states [e.g., $|9 / 2,+5 / 2\rangle$ in Fig. 1(a)], these couplings cannot lead to additional Raman transitions between $\left|g_{\uparrow, \downarrow}\right\rangle$ and other ground levels due to large two-photon detunings which are much greater than Raman fields $\left|\Omega_{0}^{2} / \Delta_{1,2}\right|[12,13,31]$, and thus are neglected. In the realistic experiment (for ${ }^{40} \mathrm{~K}$ atoms), the magnitudes of detunings $\Delta_{a, b, j}$ (can be over $1.0 \mathrm{THz}$ ) are much larger than their differences (in the order of $10 \sim 100 \mathrm{MHz}$ ) [12].
Therefore in the formulas of $V_{\sigma}$ and $M_{x, y}$ we can take that $\Delta_{1}=\Delta_{2}=\Delta_{a_{1,2}}=\Delta_{b_{1,2}}=\Delta$, which is followed by $V_{\uparrow}=V_{\downarrow}=-\left(\hbar\left|\Omega_{0}\right|^{2} / \Delta\right)\left[\cos ^{2}\left(k_{0} x\right)+\cos ^{2}\left(k_{0} y\right)\right]+$ const and $M_{0}=\hbar\left|\Omega_{0}\right|^{2} / \Delta$. Neglecting the constant terms yields the effective Hamiltonian, Eq. (1).

Before proceeding further, we provide several important remarks on the realization. First, the standing-wave lasers $\Omega_{1,2}$ are used to create both the square lattice and Raman fields, which minimizes the number of applied lasers for the realization. Second, since both the square lattice and the periodic spatial profile of the Raman fields are determined by the same standing-wave lasers, the phase fluctuations in $\Omega_{1,2}$, characterized by $\Omega_{1}=\Omega_{0} \sin \left(k_{0} x+\phi_{1}^{\text {fluc }}\right)$ and $\Omega_{2}=\Omega_{0} \sin \left(k_{0} y+\phi_{2}^{\text {fluc }}\right)$, only lead to a global shift of the lattice and Raman fields as illustrated in Fig. 1(b). The relative spatial configuration of $M_{x, y}$ and $V(\mathbf{r})$ is always automatically fixed and therefore the effective Hamiltonian, Eq. (1), is unchanged. This greatly simplifies the experimental setup in the realization. Finally, the blue detuning is essential for the realization. If using red-detuned lasers, i.e., $\Delta_{a, b, j}<0$, one has $V_{\uparrow, \downarrow}=-\left(\left|\Omega_{0}^{2} / \Delta\right|\right)\left[\sin ^{2}\left(k_{0} x\right)+\sin ^{2}\left(k_{0} y\right)\right]$, which shifts $1 / 2$ lattice site relative to the Raman fields. The reddetuned laser couplings cannot lead to 2D SO interaction or nontrivial topological orders as studied below.

The tight-binding model of $H$ can be derived straightforwardly. We take that fermions occupy the lowest $s$ orbitals $\phi_{s \sigma}(\sigma=\uparrow, \downarrow)$, and consider only the nearestneighbor hopping terms. The tight-binding Hamiltonian is given by $H_{\mathrm{QAH}}=-t_{s} \sum_{\langle\vec{i}, \vec{j}\rangle, \sigma} \hat{c}_{\vec{i} \sigma}^{\dagger} \hat{c}_{\vec{j} \sigma}+\sum_{\vec{i}} m_{z}\left(\hat{n}_{\vec{i} \uparrow}-\hat{n}_{\vec{i} \downarrow}\right)+$ $\left[\sum_{\langle\vec{i}, \vec{j} j} t_{s o} \vec{i} \vec{c}_{\vec{i} \uparrow}^{\dagger} \hat{c}_{\vec{j} \downarrow}+\right.$ H.c.], where $t_{s}$ denotes spin-conserved hopping, the 2D lattice-site index $\vec{i}=\left(i_{x}, i_{y}\right)$, and $\hat{n}_{\vec{i} \sigma}=\hat{c}_{\vec{i} \sigma}^{\dagger} \hat{c}_{\vec{i} \sigma}$. From the even parity of the $s$ orbitals and the periodic profile of Raman fields shown in Fig. 1(b), we know that the Raman fields ( $s$ orbitals) are antisymmetric (symmetric) corresponding to the center of each lattice site. Because of this relative spatial configuration, it can be directly verified that the spin-flip hopping terms due to the Raman fields satisfy $t_{\mathrm{SO}}^{j_{x}, j_{x} \pm 1}= \pm(-1)^{j_{x}} t_{\mathrm{SO}}^{(0)}$ and $t_{\mathrm{SO}}^{j_{y}, j_{y} \pm 1}=$ $\pm i(-1)^{j_{y}} t_{\mathrm{SO}}^{(0)}$, where $t_{\mathrm{SO}}^{(0)}=M_{0} \int d^{2} \vec{r} \phi_{s}(x, y) \sin \left(k_{0} x\right) \phi_{s}$ $(x-a, y)$ with $a$ the lattice constant [31]. Redefining the spin-down operator $\hat{c}_{\vec{j} \downarrow} \rightarrow e^{i \pi\left(x_{j}+y_{j}\right) / a} \hat{c}_{\vec{j} \downarrow}$, we recast the Hamiltonian into

$$
\begin{aligned}
H_{\mathrm{QAH}}= & -t_{s} \sum_{\langle\vec{i}, \vec{j}\rangle}\left(\hat{c}_{\vec{i} \uparrow}^{\dagger} \hat{c}_{\vec{j} \uparrow}-\hat{c}_{\vec{i} \downarrow}^{\dagger} \hat{c}_{\vec{j} \downarrow}\right)+\sum_{i} m_{z}\left(\hat{n}_{\vec{i} \uparrow}-\hat{n}_{\vec{i} \downarrow}\right) \\
& +\left[\sum_{j_{x}} t_{\mathrm{SO}}^{(0)}\left(\hat{c}_{j_{x} \uparrow}^{\dagger} \hat{c}_{j_{x}+1 \downarrow}-\hat{c}_{j_{x} \uparrow}^{\dagger} \hat{c}_{j_{x}-1 \downarrow}\right)+\text { H.c. }\right] \\
& +\left[\sum_{j_{y}} i t_{\mathrm{SO}}^{(0)}\left(\hat{c}_{j_{y} \uparrow}^{\dagger} \hat{c}_{j_{y}+1 \downarrow}-\hat{c}_{j_{y} \uparrow}^{\dagger} \hat{c}_{j_{y}-1 \downarrow}\right)+\text { H.c. }\right] .
\end{aligned}
$$


It can be seen that the staggered factors $(-1)^{j_{x, y}}$ in the spin-flip hopping terms effectively reverses the hopping coefficient $t_{s} \rightarrow-t_{s}$ for spin-down states, which is a key consequence of the blue-detuned laser couplings and can lead to the QAH effect [31]. In the $k$ space we have $H_{\mathrm{QAH}}=-\sum_{\mathbf{k}, \sigma \sigma^{\prime}} \hat{c}_{\mathbf{k}, \sigma}^{\dagger}\left[d_{z}(\mathbf{k}) \sigma_{z}+d_{x}(\mathbf{k}) \sigma_{x}+d_{y}(\mathbf{k}) \sigma_{y}\right]_{\sigma, \sigma^{\prime}} \hat{c}_{\mathbf{k}, \sigma^{\prime}}$, with $d_{x}=2 t_{\mathrm{SO}}^{(0)} \sin \left(k_{y} a\right), d_{y}=2 t_{\mathrm{SO}}^{(0)} \sin \left(k_{x} a\right)$ and $d_{z}=-m_{z}+$ $2 t_{s} \cos \left(k_{x} a\right)+2 t_{s} \cos \left(k_{y} a\right)$. The bulk is gapped when $\left|m_{z}\right| \neq 4 t_{s}, 0$ and the phase diagram of this Hamiltonian is determined by the first Chern number $C_{1}^{\mathrm{QAH}}=$ $(4 \pi)^{-1} \int d^{2} \mathbf{k n} \cdot \partial_{k_{x}} \mathbf{n} \times \partial_{k_{y}} \mathbf{n}$, with $\mathbf{n}=\left(d_{x}, d_{y}, d_{z}\right) /|\vec{d}(\mathbf{k})|$. In particular, one can verify that $C_{1}^{\mathrm{QAH}}=\operatorname{sgn}\left(m_{z}\right)$ when $0<\left|m_{z}\right|<4 t_{s}$ and $t_{\text {SO }}^{(0)} \neq 0$, and the above Hamiltonian describes a quantum anomalous Hall insulator [16]. On the other hand, for the regime with $\left|m_{z}\right|>4 t_{s}$ the system is a trivial insulator.

We next study the topological chiral superfluid phase by considering an $s$-wave interaction which can be well controlled with Feshbach resonance in cold atoms [32]. In an optical lattice, this interaction is described by the attractive Fermi Hubbard model $H_{\text {int }}=-\sum_{\vec{i}} U n_{\vec{i} \uparrow} n_{\vec{i} \downarrow}$, where the regularized 2D effective interaction $U=\hbar^{2} a_{s} \sqrt{8 \pi} /\left(m l_{z} l_{2 \mathrm{~d}}^{2}\right)$ with $l_{z}=\left(h / m \omega_{z}\right)^{1 / 2}, l_{2 \mathrm{D}}=\left(h / m \omega_{2 D}\right)^{1 / 2}$, and the $s$-wave scattering length $a_{s}>0$. The parameters $\omega_{2 \mathrm{D}}$ and $\omega_{z}$ denote the $2 \mathrm{D}$ square lattice trapping frequency and a tight trapping frequency along the $z$ axis, respectively. We first study the superfluid phase with the self-consistent mean-field approach by introducing the $s$-wave superfluid order parameter by $\Delta_{s}=\left(U / N_{0}\right) \sum_{\mathbf{k}}\left\langle c_{\mathbf{k} \uparrow} c_{-\mathbf{k} \downarrow}\right\rangle$ with $N_{0}$ the number of lattice sites. Then the Bogoliubov de Gennes $(\mathrm{BdG})$ Hamiltonian in the Nambu basis $\psi_{\mathbf{k}}=$ $\left(c_{\mathbf{k} \uparrow}, c_{\mathbf{k} \downarrow}, c_{-\mathbf{k} \downarrow}^{\dagger},-c_{-\mathbf{k} \uparrow}^{\dagger}\right)$ can be written as $H_{\mathrm{BdG}}=$ $\sum_{\mathbf{k}} \psi_{\mathbf{k}}^{\dagger} \mathcal{H}_{\mathrm{BdG}}(\mathbf{k}) \psi_{\mathbf{k}}$, where

$$
\begin{aligned}
\mathcal{H}_{\mathrm{BdG}}= & d_{x} \sigma_{x} \otimes \tau_{z}+d_{y} \sigma_{y} \otimes \tau_{z}+d_{z} \sigma_{z} \otimes I-\mu I \otimes \tau_{z} \\
& +\left(\Delta_{s} I \otimes \tau_{+}+\text {H.c. }\right) .
\end{aligned}
$$

Here $\mu$ is the chemical potential, $\tau_{x, y, z}$ are Pauli matrices acting on the Nambu space, and $\tau_{ \pm}=\left(\tau_{x} \pm i \tau_{y}\right) / 2$. The $s$-wave order parameter is solved by the gap equation

$$
\frac{N_{0}}{U}=\frac{1}{4} \sum_{\mathbf{k}, \alpha= \pm} \frac{1}{E_{\mathbf{k}}^{\alpha}}\left[1+\alpha \frac{d_{z}^{2}(\mathbf{k})}{w_{\mathbf{k}}}\right] \tanh \left(\frac{1}{2} \beta E_{\mathbf{k}}^{\alpha}\right),
$$

where the energy spectra $E_{\mathbf{k}}^{ \pm}=\left(\Gamma^{2}+\left|\vec{d}_{\mathbf{k}}\right|^{2} \pm 2 w_{\mathbf{k}}\right)^{1 / 2}$ by diagonalizing $\mathcal{H}_{\mathrm{BdG}}$, with $w_{\mathbf{k}}=\left[\mu^{2}\left(d_{x}^{2}+d_{y}^{2}\right)+d_{z}^{2} \Gamma^{2}\right]^{1 / 2}$ and $\Gamma^{2}=\mu^{2}+\left|\Delta_{s}\right|^{2}$, and $\beta=1 /\left(k_{B} T\right)$.

To identify an effective $p+i p$ chiral superfluid phase from the Hamiltonian $\mathcal{H}_{\mathrm{BdG}}$, we analyze the special situation with $m_{z}=4 t_{s}$. In this case, the low-energy spectrum is captured by the Hamiltonian (up to linear order of the momentum) $\mathcal{H}_{\mathrm{BdG}}=2 t_{\mathrm{SO}}^{(0)}\left(k_{x} \sigma_{y}+k_{y} \sigma_{x}\right) \tau_{z}$ $-\mu \tau_{z}+\left(\Delta_{s} \tau_{+}+\right.$H.c. $)$. It is interesting that this low-energy model is equivalent to the surface Hamiltonian of a threedimensional topological insulator in proximity to an $s$-wave SC [4], which renders the 2D chiral $p+i p$ topological SC phase. We therefore expect that in our model the chiral Majorana edge modes and non-Abelian MZM in the vortex core can be obtained [33]. The full SF phase diagram can be determined by analyzing the properties of the bulk gap. By examining $E_{\mathbf{k}}^{-}$with $m_{z}>0$ we find that the bulk gap closes at $\mathbf{k}=(0,0)$ and $\mathbf{k}=(\pi, \pi)$ for $\mu^{2}+\left|\Delta_{s}\right|^{2}=\left(m_{z}-4 t_{s}\right)^{2}$ and $\left(m_{z}+4 t_{s}\right)^{2}$, respectively. Furthermore, at the points $\mathbf{k}=(0, \pi)$ and $\mathbf{k}=(\pi, 0)$ the bulk gap closes if $\mu^{2}+\left|\Delta_{s}\right|^{2}=m_{z}^{2}$. These properties lead to a rich phase diagram with $t_{\mathrm{SO}}^{(0)} \neq 0$ as presented below. From the lowenergy Hamiltonian $\mathcal{H}_{\mathrm{BdG}}$ we know that the superfluid phase with $\left|\Delta_{s}\right| \gtrsim 0, m_{z}=4 t_{s}$, and $\mu=0$ is topological. This implies that if $\left|m_{z}\right|>2 t_{s}$, the SF phase is topologically nontrivial with Chern number $C_{1}^{\mathrm{SF}}=+1$ for $\left(\left|m_{z}\right|-4 t_{s}\right)^{2}<\mu^{2}+\left|\Delta_{s}\right|^{2}<m_{z}^{2}$, and $C_{1}^{\mathrm{SF}}=-1$ for $m_{z}^{2}<$ $\mu^{2}+\left|\Delta_{s}\right|^{2}<\left(\left|m_{z}\right|+4 t_{s}\right)^{2}$. The change by two in the Chern number originates from the fact that tuning $\mu^{2}+\left|\Delta_{s}\right|^{2}$ from less than to larger than $m_{z}^{2}$ reverses the mass terms at both $\mathbf{k}=(0, \pi)$ and $\mathbf{k}=(\pi, 0)$. On the other hand, we know that when $0<m_{z}<2 t_{s}$ and $\mu^{2}+\left|\Delta_{s}\right|^{2}<\left|m_{z}\right|^{2}$ the system is in the QAH insulating phase with $C_{1}^{\mathrm{QAH}}=1$ or equivalently $C_{1}^{\mathrm{SF}}=+2$, since the Nambu space doubles the Chern number. Starting from this QAH phase and tuning the parameters into the regime, $m_{z}^{2}<\mu^{2}+\left|\Delta_{s}\right|^{2}<\left(\left|m_{z}\right|-4 t_{s}\right)^{2}$ with $\left|m_{z}\right|<2 t_{s}$ reverses the Dirac mass terms at $\mathbf{k}=(0, \pi)$ and $\mathbf{k}=(\pi, 0)$, yielding a trivial phase in this parameter regime. Finally, for the parameter regime with $\left(\left|m_{z}\right|-4 t_{s}\right)^{2}<$ $\left.\mu^{2}+\left|\Delta_{s}\right|^{2}<\left|m_{z}\right|+4 t_{s}\right)^{2}$ and $\left|m_{z}\right|<2 t_{s}$, one further gets that $C_{1}^{\mathrm{SF}}=-1$. In cold atoms the parameters such as $m_{z}, t_{s}$ and $\mu$ can be precisely adjusted in the experiment to tune the system into topological chiral SF phases.

The self-consistent solutions for $\Delta_{s}$ and the mean-field phase diagram are shown in Figs. 2(a) and (b), respectively. It can be seen that the $s$-wave order $\Delta_{s}$ vanishes when the noninteracting Hamiltonian $H_{\mathrm{TI}}$ has a large insulating gap and the chemical potential $\mu$ is located deep in the band gap [left most area in (a)]. On the other hand, an appreciable $\Delta_{s}$ is obtained in the area with $1.5 t_{s}<\mu<3.5 t_{s}$ and $0<m_{z}<3.0$. In such a parameter regime the SO terms of $d_{x}$ and $d_{y}$ dominate over $d_{z}$ and a relatively large density of states at the Fermi level is present. In Fig. 2(b) the superfluid phases with both $C_{1}^{\mathrm{SF}}=+1$ and -1 are obtained, respectively, leading to chiral and antichiral Majorana edge modes localized in the boundary [1].

Note that in 2D superfluids no long-range order exists at the finite temperature and the critical temperature by meanfield theory is often overestimated. Instead, the BerezinskyKosterlitz-Thouless (BKT) transition occurs at the critical temperature which is limited by entropically driven vortex 

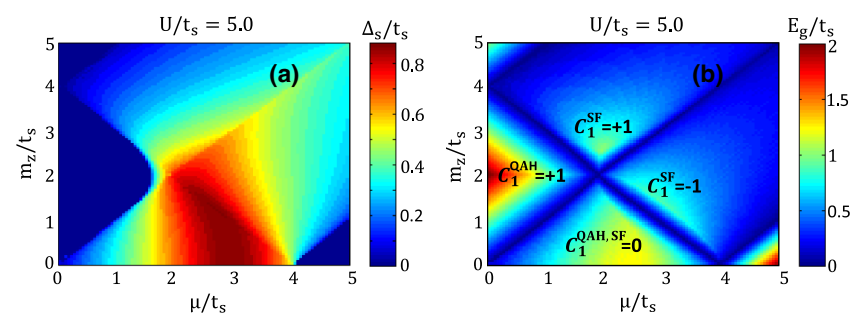

FIG. 2 (color online). Self-consistent calculations of the $s$-wave order $\Delta_{s}$ [for (a)] and the bulk gap $E_{g}$ [for (b)] versus $m_{z}$ and $\mu$ at zero temperature and with $U=5 t_{s}$. Topological phase transition occurs at $E_{g}=0$. The hopping coefficients $t_{\text {SO }}^{(\sigma)}=t_{s}$. In plotting (b) the gap $E_{g}$ in the QAH regime $\left(C_{1}^{\mathrm{QAH}}=+1\right)$ is reduced by half.

and antivortex proliferation [34]. The BKT temperature is calculated by

$$
T_{\mathrm{BKT}}=\frac{\pi}{2} \rho_{s}\left(\Delta_{s}, T_{\mathrm{BKT}}\right),
$$

where $\rho_{s}$ is the superfluid stiffness (superfluid density). In the presence of the phase fluctuation, the superfluid order parameter takes the form $\Delta_{s}=\Delta_{0} e^{i \theta(\mathbf{r})}$, where $\theta$ varies slowly in the position space. The simplest way to estimate the stiffness is to use its relation to the supercurrent density $\mathbf{j}_{s}=\rho_{s} \nabla \theta(\mathbf{r})$. For the case that $\theta$ varies slowly with the position, we can approximate that $\mathbf{q}=\nabla \theta(\mathbf{r})$, which implies that the $s$-wave pairing occurs between two fermions with a center-of-mass momentum q. The current can also be calculated by the variation of the free energy $\mathbf{j}_{s}=$ $\frac{1}{A} \operatorname{Tr}\left[e^{-\beta H_{\mathrm{BdG}}} \delta H_{\mathrm{BdG}}\left(\mu, \Delta_{0}, \nabla \theta\right) / \delta \mathbf{q}\right] / \operatorname{Tr}\left[e^{-\beta H_{\mathrm{BdG}}}\right]$, with $A$ the area of the lattice. Solving this equation and the gap equation for $\Delta_{s}$, together we can get the stiffness by $\rho_{s}=\mathbf{j}_{s} / \mathbf{q}$. It can be verified that these results are consistent with those through a standard functional path integral approach, where the phase fluctuation of the superfluid order is described by an effective action $S_{\text {fluc }}=(1 / 2) \int d^{2} \mathbf{r} \rho_{s}(\nabla \theta)^{2}$. The BKT temperature is then given by Eq. (5). We have numerically confirmed that when $\Delta_{s}=0$ the supercurrent $\mathbf{j}_{s}$ vanishes.

The numerical results for $T_{\text {BKT }}$ versus $U$ and $\mu$ are shown in Fig. 3. For the case that the Fermi energy touches the bulk edge, the BKT temperature increases gradually with $U$ [blue line in Fig. 3(a)], while when the Fermi energy is located deep into the bulk, the $T_{\mathrm{BKT}}$ increases sharply with $U$ and approaches a saturate magnitude in a small Hubbard interaction (red, green, and black curves). The BKT temperature versus $\mu$ with fixed $U=5 t_{s}$ and $m_{z}$ is plotted in Fig. 3(b). It can be found that with $m_{z}=3 t_{s}$, the maximum $T_{\mathrm{BKT}}$ is around $0.3 t_{s}$. Note that the upper value of the blue detuning is limited by the fine-structure splitting and can be taken as $2 \pi \times 1.7 \mathrm{THz}$ for ${ }^{40} \mathrm{~K}$ atoms [12,35], which gives the recoil energy $E_{R} / \hbar \sim \hbar k_{0}^{2} / 2 m=$ $2 \pi \times 8.5 \mathrm{kHz}$ using lasers of wavelength $764 \mathrm{~nm}$ to form the square lattice. Taking $\Omega_{0}=2 \pi \times 0.27 \mathrm{GHz}$, we have $V_{0}=5 E_{R}$, the lattice trapping frequency $\omega=2 \pi \times 34.9 \mathrm{kHz}$, and the hopping coefficients (a)
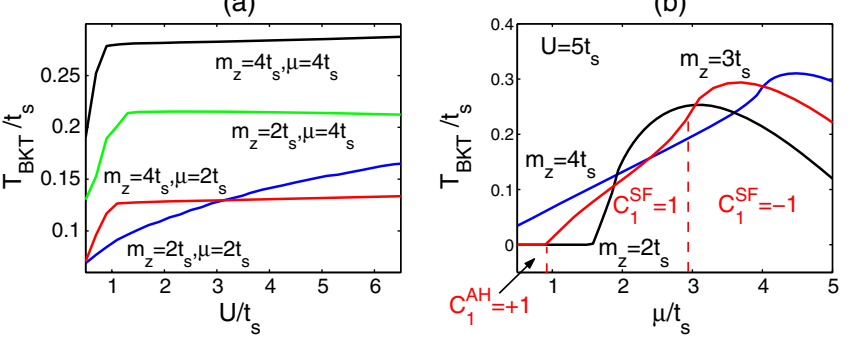

FIG. 3 (color online). BKT temperature as a function of $U$ with different values of $m_{z}$ and $\mu$ (a), and versus $\mu$ at $U=5 t_{s}$ and with different magnitudes of $m_{z}$ (b). The SO coupled hopping $t_{\mathrm{SO}}^{(0)}=t_{s}$. Topological orders of different Chern numbers are indicated for the case with $m_{z}=3 t_{s}$.

$t_{s o}^{(0)} / \hbar \sim t_{s} / \hbar \simeq 2 \pi \times 0.52 \mathrm{kHz}$. With this parameter regime we find that the bulk gap for QAH state $E_{g}=$ $2 \pi \times 2.08 \mathrm{kHz}$ by setting $\delta=4 t_{s}$, corresponding to the temperature $T \approx 0.33 T_{F} \approx 100 \mathrm{nK}$ for observation, and $T_{\mathrm{BKT}}$ for the topological superfluid phase with $U=5 t_{s}$, $\delta=6 t_{s}$, and $\mu=-3.5 t_{s}$ is about $0.086 T_{F}$.

Before concluding we provide further discussions on the experimental studies of our proposal. Note that for the bluedetuned optical lattice, the atoms are trapped in the minima of lattice potentials which may generally minimize heating effects [36]. With the previous parameter regime for ${ }^{40} \mathrm{~K}$ atoms (with a natural linewidth $\Gamma \approx 2 \pi \times 6.04 \mathrm{MHz}$ ) the lifetime can be estimated to be $\tau \approx 4 \Delta /(\omega \Gamma) \approx 5.1 \mathrm{~s}$ [37], which is long enough for experimental detections. On the other hand, while the topological superfluid phase with Majorana modes requires a fermion system, the SO coupling and QAH states can be demonstrated with cold bosons [38], which makes such experimental observations be even more promising. It is worthwhile to point out that 2D chiral topological orders are classified by a $Z$ invariant, and are stable when stacking multilayers of the 2D system. This enables a study of topological superfluid in the many-layer square lattice system which should have a higher transition temperature since the fluctuation in the superfluid order can be suppressed.

In conclusion, we have proposed a truly experimental scheme to observe 2D SO interaction which leads to both the quantum anomalous Hall effect and chiral topological superfluid phase for cold atoms trapped with a bluedetuned square optical lattice and coupled to two periodic Raman fields. We introduced a new technique by integrating the creations of optical lattice and Raman fields through the same standing-wave lasers, which greatly simplifies the experimental setup and leads to the minimal realization of the 2D SO interaction and exotic topological phases. We calculated the bulk gap of the topological states and the BKT temperature of the topological superfluid phase, and show that the predicted topological orders are reachable with the realistic parameter regimes. The feasibility of our proposal will motivate future experimental studies on the 2D topological orders and the observation of non-Abelian Majorana modes with realistic cold atom platforms. 
We appreciate Gyu-boong Jo, Waseem Bakr, Lawrence Cheuk, and Shuai Chen much for very helpful discussions on the experimental feasibility of this work. We also thank Wujie Huang, H. Zhai, M. Cheng, Patrick A. Lee, C. Wu, and Z.-X. Liu for insightful discussions. We acknowledge the support from HKRGC through Grants No. 605512 and No. HKUST3/CRF09.

[1] N. Read and D. Green, Phys. Rev. B 61, 10267 (2000); A. Kitaev, Phys. Usp. 44, 131 (2001).

[2] D. A. Ivanov, Phys. Rev. Lett. 86, 268 (2001); J. Alicea, Y. Oreg, G. Refael, F. von Oppen, and M. R. A. Fisher, Nat. Phys. 7, 412 (2011).

[3] S. Das Sarma, M. Freedman, and C. Nayak, Phys. Rev. Lett. 94, 166802 (2005).

[4] L. Fu and C. L. Kane, Phys. Rev. Lett. 100, 096407 (2008); X.-L. Qi, T. L. Hughes, and S.-C. Zhang, Phys. Rev. B 82, 184516 (2010).

[5] J. D. Sau, R. M. Lutchyn, S. Tewari, and S. Das Sarma, Phys. Rev. Lett. 104, 040502 (2010).

[6] R. M. Lutchyn, J. D. Sau, and S. Das Sarma, Phys. Rev. Lett. 105, 077001 (2010); Y. Oreg, G. Refael, and F. von Oppen, ibid. 105, 177002 (2010); A. C. Potter and P. A. Lee, ibid. 105, 227003 (2010).

[7] V. Mourik, K. Zuo, S. M. Frolov, S. R. Plissard, E. P. A. M. Bakkers, and L.P. Kouwenhoven, Science 336, 1003 (2012); M. T. Deng, C. L. Yu, G. Y. Huang, M. Larsson, P. Caroff, and H. Q. Xu, Nano Lett. 12, 6414 (2012); Y. Ronen, Y. Most, Y. Oreg, M. Heiblum, and H. Shtrikman, Nat. Phys. 8, 887 (2012).

[8] K. T. Law, P. A. Lee, and T. K. Ng, Phys. Rev. Lett. 103, 237001 (2009); K. Flensberg, Phys. Rev. B 82, 180516(R) (2010); X.-J. Liu and A. M. Lobos, ibid. 87, 060504(R) (2013).

[9] X.-J. Liu, M. F. Borunda, X. Liu, and J. Sinova, Phys. Rev. Lett. 102, 046402 (2009).

[10] Y.-J. Lin, K. Jiménez-García, and I. B. Spielman, Nature (London) 471, 83 (2011).

[11] M. Chapman and C. Sá de Melo, Nature (London) 471, 41 (2011).

[12] P. Wang, Z.-Q. Yu, Z. Fu, J. Miao, L. Huang, S. Chai, H. Zhai, and J. Zhang, Phys. Rev. Lett. 109, 095301 (2012).

[13] L. W. Cheuk, A. T. Sommer, Z. Hadzibabic, T. Yefsah, W. S. Bakr, and M. W. Zwierlein, Phys. Rev. Lett. 109, 095302 (2012).

[14] J.-Y. Zhang et al., Phys. Rev. Lett. 109, 115301 (2012).

[15] X.-J. Liu, X. Liu, L. C. Kwek, and C. H. Oh, Phys. Rev. Lett. 98, 026602 (2007); Phys. Rev. B 79, 165301 (2009).

[16] C. Wu, Phys. Rev. Lett. 101, 186807 (2008); X.-J. Liu, X. Liu, C. Wu, and J. Sinova, Phys. Rev. A 81, 033622 (2010); M. Zhang, H.-h. Hung, C. Zhang, and C. Wu, ibid. 83, 023615 (2011); N. Goldman, J. Beugnon, and F. Gerbier, Phys. Rev. Lett. 108, 255303 (2012); S.-S. Zhang, H. Fan, and W.-M. Liu, Phys. Rev. A 87, 023622 (2013).

[17] N. Goldman, I. Satija, P. Nikolic, A. Bermudez, M. A. Martin-Delgado, M. Lewenstein, and I. B. Spielman, Phys.
Rev. Lett. 105, 255302 (2010); N. Goldman, J. Beugnon, and F. Gerbier, ibid. 108, 255303 (2012).

[18] L.-J. Lang, X. Cai, and S. Chen, Phys. Rev. Lett. 108, 220401 (2012).

[19] X.-J. Liu, Z.-X. Liu, and M. Cheng, Phys. Rev. Lett. 110, 076401 (2013); X. Li, E. Zhao, and W. V. Liu, Nat. Commun. 4, 1523 (2013).

[20] J. Ruostekoski, G. V. Dunne, and J. Javanainen, Phys. Rev. Lett. 88, 180401 (2002).

[21] C. Zhang, S. Tewari, R. M. Lutchyn, and S. Das Sarma, Phys. Rev. Lett. 101, 160401 (2008); Y. Zhang, L. Mao, and C. Zhang, ibid. 108, 035302 (2012).

[22] M. Sato, Y. Takahashi, and S. Fujimoto, Phys. Rev. Lett. 103, 020401 (2009); S.-L. Zhu, L. B. Shao, Z. D. Wang, and L.-M. Duan, Phys. Rev. Lett. 106, 100404 (2011).

[23] J. D. Sau, R. Sensarma, S. Powell, I. B. Spielman, and S. Das Sarma, Phys. Rev. B 83, 140510(R) (2011).

[24] X.-J. Liu and P. D. Drummond, Phys. Rev. A 86, 035602 (2012); R. Wei and E. J. Mueller, ibid. 86, 063604 (2012); X.-J. Liu, ibid. 87, 013622 (2013).

[25] J. P. Vyasanakere, and V. B. Shenoy, Phys. Rev. B 83, 094515 (2011); J. P. Vyasanakere S. Zhang, and V. B. Shenoy, ibid. 84, 014512 (2011).

[26] W. Yi and G.-C. Guo, Phys. Rev. A 84, 031608(R) (2011); L. He and X.-G. Huang, Phys. Rev. Lett. 108, 145302 (2012); F. Wu, G.-C. Guo, W. Zhang, and W. Yi, ibid. 110, 110401 (2013).

[27] Z.-Q. Yu and H. Zhai, Phys. Rev. Lett. 107, 195305 (2011); H. Hu, L. Jiang, X.-J. Liu, and H. Pu, ibid. 107, 195304 (2011); L. Dell'Anna, G. Mazzarella, and L. Salasnich, Phys. Rev. A 86, 053632 (2012).

[28] Y. Li, L. P. Pitaevskii, and S. Stringari, Phys. Rev. Lett. 108, 225301 (2012); K. Seo, L. Han, and C. A. R. Sá de Melo, ibid. 109, 105303 (2012).

[29] D.-W. Zhang, Z.-Y. Xue, H. Yan, Z. D. Wang, and S.-L. Zhu, Phys. Rev. A 85, 013628 (2012); M.-Y. Ye and X.-M. Lin, arXiv:1207.5369v1.

[30] V. Galitski and I. B. Spielman, Nature (London) 494, 49 (2013); X. Zhou, Y. Li, Z. Cai, and C. Wu, J. Phys. B 46, 134001 (2013).

[31] See the Supplemental Material at http://link.aps.org/ supplemental/10.1103/PhysRevLett.112.086401 for more details.

[32] I. Bloch, J. Dalibard, and W. Zwerger, Rev. Mod. Phys. 80, 885 (2008).

[33] S. Tewari, S. Das Sarma, C. Nayak, C. Zhang, and P. Zoller, Phys. Rev. Lett. 98, 010506 (2007).

[34] V. L. Berezinskii, Sov. Phys. JETP 32, 493 (1971); J. M. Kosterlitz and D. Thouless, J. Phys. C 5, L124 (1972).

[35] T. G. Tiecke, Ph.D. thesis, University of Amsterdam, 2009.

[36] R. Ozeri, L. Khaykovich, and N. Davidson, Phys. Rev. A 59, R1750 (1999); F. Gerbier and Y. Castin, ibid. 82, 013615 (2010); M. Takamoto, H. Katori, S. I. Marmo, V. D. Ovsiannikov, and V. G. Pal'chikov, Phys. Rev. Lett. 102, 063002 (2009).

[37] D. Jaksch and P. Zoller, Ann. Phys. (Amsterdam) 315, 52 (2005).

[38] M. Atala, M. Aidelsburger, J. T. Barreiro, D. Abanin, T. Kitagawa, E. Demler, and I. Bloch, arXiv:1212.0572v1. 